\title{
DIFUSÃO DA COVID-19 NAS GRANDES ESTRUTURAS TERRITORIAIS DO ESTADO DO RIO GRANDE DO SUL, BRASIL
}

\section{DIFFUSION OF COVID-19 IN THE GREAT SPACE STRUCTURES OF THE STATE OF RIO GRANDE DO SUL, BRAZIL}

\author{
Rivaldo Faria \\ Doutor em Geografia (IG/UNICAMP) \\ Prof. Adjunto da Universidade Federal de Santa Maria \\ rivaldofaria.geo@gmail.com \\ Douglas Bouvier Erthal \\ Mestrando em Geografia, Universidade Federal de Santa Maria \\ douglasbouv@gmail.com \\ lago Turba Costa \\ Doutorando em Geografia, Universidade Federal de Santa Maria \\ iagoturba06@gmail.com \\ Maurício Rizzatti \\ Doutorando em Geografia, Universidade Federal de Santa Maria \\ geo.mauricio.rizzatti@gmail.com \\ Pedro Leonardo Cezar Spode \\ Doutorando em Geografia, Universidade Federal de Santa Maria \\ pedrospode@gmail.com
}

\begin{abstract}
RESUMO
O presente estudo tem como objetivo analisar a difusão espacial da COVID-19 e caracterizá-la em relação às grandes estruturas territoriais do estado do Rio Grande do Sul. Trata-se de um estudo ecológico, realizado com todos os casos registrados de COVID-19 (infectados e óbitos), no período de 10 de março à 02 de maio de 2020. Os dados foram coletados em fontes secundárias oficiais e modelados por município de residência do caso. A difusão dos casos e sua densificação permitiu reconhecer dois grandes eixos de difusão da COVID-19 no estado do Rio Grande do Sul: na rede urbana que vai de Porto Alegre à serra gaúcha, que é a rede de maior densidade e de maior fluxo, e na rede que vai na direção da campanha, que é uma rede urbana de menor densificação e fluxo, mais antiga e também mais fragmentada. Por essa razão, é maior o número de casos na rede urbana da serra, estendendo-se, por conseguinte, até Passo Fundo e Erechim. Mais de $90 \%$ dos casos estão concentrados em municípios de baixa privação social e melhores condições de saúde, exatamente no eixo Porto Alegre-Serra-Norte gaúcho. Todavia, há sinais de interiorização da COVID-19 em municípios de elevada privação social, sobretudo os localizados na região do Pampa e nas fronteiras sul e oeste. Recomenda-se, dessa forma, enorme atenção à interiorização do vírus, seja porque a extensão geográfica do evento torna mais difícil o seu controle, seja ainda porque municípios socialmente mais privados possuem piores condições de saúde e maior dificuldade de acesso aos serviços de saúde.
\end{abstract}

Palavras-chave: Coronavírus. Difusão Espacial. Privação Social. Rede urbana.

\begin{abstract}
The present study aims to analyze the spatial diffusion of COVID-19 and characterize it in relation to the territorial structures of the state of Rio Grande do Sul. It is an ecological study, carried out with all registered cases of COVID-19 (infected and deaths), from March 10 to May 2, 2020. The data were collected from official secondary sources and modeled by municipality of residence of the case. The diffusion of the cases and their densification made it possible to recognize two major axes of diffusion of COVID-19 in the state of Rio Grande do Sul: in the urban network that goes from Porto Alegre to the Serra Gaúcha, which is the network with the highest density and highest flow, and in the network that goes towards the Campanha, which is an urban network with less densification and flow, older and also more fragmented. For this reason, the number of cases in the urban network of the Serra Gaúcha is greater, extending, therefore, to Passo Fundo and Erechim. More than $90 \%$ of the cases are concentrated in municipalities with low social deprivation
\end{abstract}

Recebido em: 08/05/2020

Aceito para publicação em: 21/05/2020. 
and better health conditions, exactly on the Porto Alegre-Serra-North axis of the state. However, there are signs of COVID-19 interiorization in municipalities with high social deprivation, especially those located in the Pampa region and on the southern and western borders. Thus, it is recommended that enormous attention be paid to the diffusion of the virus, either because the geographic extent of the event makes it more difficult to control, or because socially more private municipalities have worse health conditions and greater difficulty in accessing health services.

Keywords: Coronavírus. Spatial diffusion. Social Deprivation. Urban Network.

\section{INTRODUÇÃO}

Em dezembro de 2019, alguns casos de pneumonia de origem desconhecida foram detectados em Wuhan, capital da província de Hubei, na China (GUAN et.al, 2020). Descobriu-se, então, que se tratava de um novo coronavírus, conhecido por causar infecções respiratórias agudas e graves (LI e LIN, 2013). A Organização Mundial de Saúde (OMS), observando sua rápida disseminação pelo mundo, acabou definindo a SARSCoV-2 (severe acute respiratory syndrome coronavirus) ou COVID-19 como uma pandemia (WHO, 2020), ou seja, um evento epidêmico que acontece simultaneamente em diversos países do mundo. No Brasil, o primeiro caso confirmado pelo vírus foi registrado em 26 de fevereiro de 2020, na cidade de São Paulo, e até o dia 30 de abril de 2020, o país já havia 96.396 casos confirmados espalhados pelas suas 27 Unidades Federativas, com o total de 6.724 óbitos (BRASIL, 2020).

Pelas dimensões e gravidade do vírus e pela realidade do mundo globalizado do século XXI, a COVID-19 é um evento de enorme complexidade. Razão porque não só as ciências médicas, mas ciências, em geral, estão sendo requisitadas nesse momento de pandemia. É exemplo as ciências estatísticas e matemáticas, no que tange as análises probabilísticas e de tendências de disseminação do vírus pelas cidades e países, como também as ciências humanas, como a sociologia e a geografia. A geografia, em especial, está em contato com os problemas de saúde desde antes de John Snow, que produziu a cartografia da distribuição espacial da cólera em Londres, no ano de 1854. No estágio científico e moderno da geografia, a preocupação com a saúde esteve presente em obras seminais, como nos trabalhos de Elisée Reclus e Max Sorre. A Max Sorre, inclusive, devemos o conceito de "complexos patogênicos" (SORRE, 1984), talvez importante para compreensão da COVID-19 nesse momento.

A geografia tem papel de destaque na discussão e proposições de estratégias acerca de eventos epidêmicos, visto que diante da inexistência de alguma vacina, a estratégia mais adequada e recomendada pela OMS e pelos especialistas na área da saúde é o isolamento social (PORSSE et.al, 2020). Trata-se, naturalmente, de uma estratégia geográfica usada para restringir a fluidez das pessoas pelo território, impedindo, dessa forma, que o vírus se dissemine ainda mais. Para além disso, ela também oferece uma série de técnicas e conceitos que nos ajudam a compreender a dinâmica da epidemia. Uma característica importante dos primeiros casos confirmados no Brasil é que essas pessoas infectadas, que retornaram de viagens internacionais, pertencem às classes sociais mais abastadas (FARIAS, 2020), ou seja, é um vírus que inicialmente se dissemina através de pessoas de classe média e classe média-alta, para, posteriormente, se alastrar nas periferias das cidades. Por isso, em relação ao território, o vírus se expande mais rapidamente nos centros de maior densidade e fluidez econômica. Um primeiro estudo feito por Spósito e Guimarães (2020) demostrou, por exemplo, que o vírus vem se disseminando por meio da rede urbana, primeiro nas metrópoles, logicamente, e no segundo momento, avançando para as cidades médias e pequenas.

É fundamental, portanto, reconhecer a dinâmica espacial da COVID-19 nas estruturas espaciais de cada região e estado, porque eles diferem e podem conduzir a desafios particulares para o controle da epidemia. Este é o primeiro objetivo deste estudo, a ser aplicado para a compreensão da dinâmica espacial da COVID19 no estado do Rio Grande do Sul. Um segundo objetivo é analisar essa dinâmica espacial em relação às condições sociais dos municípios através de um indicador multicritério de privação social. Essa é uma forma de fazer pensar sobre os desafios de planejar a epidemia em relação às diferentes condições sociais das regiões e municípios gaúchos.

\section{MATERIAIS E MÉTODOS}

Este é um estudo ecológico realizado com todos os casos registrados de COVID-19 (infectados e óbitos) no período de 10 de março à 02 de maio de 2020. Os dados foram coletados na plataforma "Brasil.IO" 
(https://brasil.io/home/), ao nível dos municípios do estado do Rio Grande do Sul e modelados por local de residência do caso.

A avaliação da evolução espacial dos casos foi feita por semanas epidemiológicas e as análises espaciais foram realizadas com uso de técnicas exploratórias e avaliação através do estimador de densidade de casos de Kernel, conforme orientação de Camargo, Fucks e Câmara (2004). Quanto aos parâmetros usados no estimador de Kernel, foi aplicado um raio de influência de 120 quilômetros, que foi o valor calculado usandose um critério mínimo de vizinhança (10) e a extensão do estado do Rio Grande do Sul $\left(281748 \mathrm{~km}^{2}\right)$. O método usado é o bidimensional uniforme, que pondera os valores da vizinhança em relação a uma dada área e cujo fator é a distância. Com esses recursos foi possível identificar adensamentos/agrupamentos e as rotas de dispersão do evento, o que permitiu ainda classificar grandes áreas de difusão do vírus e possíveis áreas em processo de evolução.

O Índice de Privação Social (IPS) foi elaborado para compreender a difusão da COVID-19 em relação aos estratos populacionais e de municípios conforme as diferentes condições sociais. O IPS é um modelo de avaliação multicritério criado a partir de dados selecionados do Atlas do Desenvolvimento Humano (ADH, 2013) e do Instituto Brasileiro de Geografia e Estatística (IBGE, 2010). A primeira etapa para elaboração do modelo multicritério é a seleção das variáveis que irão compô-lo. Para realizá-la foram realizadas duas rodadas de avaliação pelos autores deste trabalho. A primeira avaliou a capacidade do indicador para medir a privação de condições de vida, tendo em vista as dimensões saúde, renda, educação e pobreza. Isso levou à seleção de dez categorias que foram elaboradas e espacializadas no mapa. A segunda rodada tratou de avaliar a representatividade do indicador para os 497 municípios, assim como a fiabilidade em termos de variação ou oscilação aleatória. Dessa segunda rodada foram restaram seis indicadores que foram inseridos no modelo (quadro 1)

\begin{tabular}{|l|l|l|}
\hline \multicolumn{2}{|l|}{ Quadro 1 - Indicadores sociais elaborados para o Índice de Privação Social dos municípios do Estado } \\
do Rio Grande do Sul. \\
\hline Dimensões & Relação dos indicadores & Fonte \\
\hline \multirow{2}{*}{ Renda } & Percentual da renda apropriada pelos 40\% mais pobres 2010 & ADH \\
\cline { 2 - 3 } & Razão de dependência 2010 & ADH \\
\hline Saúde & Esperança de vida ao nascer 2010 & ADH \\
\hline Educação & Taxa de analfabetismo - 15 anos ou mais 2010 & ADH \\
\hline \multirow{2}{*}{ Pobreza } & $\%$ de vulneráveis à pobreza 2010 & ADH \\
\cline { 2 - 3 } & $\%$ população inativa com renda igual ou inferior a 1/2 salário & IBGE \\
\hline \multicolumn{2}{|c|}{ Fonte: (Altas do Desenvolvimento Humo, 2013) (IBGE 2010) } \\
\hline
\end{tabular}

A segunda etapa do modelo é a elaboração dos indicadores selecionados e sua padronização. Isso é necessário porque os indicadores têm, cada um à sua maneira, uma escala diferente de medida. A padronização, portanto, é um recurso usado para colocar todos os indicadores numa mesma escala. A técnica usada para padronização neste trabalho é a normalização através dos valores máximo e mínimo, que é a usada na elaboração dos indicadores do Índice de Desenvolvimento Humano, de modo que todos os indicadores foram padronizados numa escala de 0 a 1 . Entretanto, por se tratar da avaliação da privação, ou seja, da ausência de condições, invertemos a escala para que valores próximos de um indiquem elevada privação e próximos de zero o contrário.

A terceira etapa, então, é a ponderação dos indicadores, ou seja, a técnica de atribuir pesos de importâncias diferentes aos indicadores, conforme suas capacidades de medir ou avaliar a privação social. Para fazer isso utilizamos o Método de Análise Hierárquica (Analytic Hierarchy Process), (AHP), conforme orientação de Saaty (1990) e Costa (2002). Por esse método os indicadores foram submetidos a uma matriz de comparação par a par e hierarquizados, de modo que a cada um foi recebeu, ao final, um peso (weight) refletido num valor de importância do indicador.

A quarta etapa, finalmente, é a combinação dos critérios (indicadores) padronizados e ponderados. Para fazer isso foi usado a técnica da combinação linear combinada (WLC), que combina os fatores pode meio de uma média ponderada, conforme orientação de Voogd (1983).

As análises foram realizadas com uso dos os softwares ARCGIS 10.8 e QGIS 3.12.0 e a tabulação foi feita no Excel do pacote Office.

\section{RESULTADOS E DISCUSSÃO}

O primeiro caso de COVID-19 no estado do Rio Grande do Sul foi registrado no dia 10 de março de 2020 e até 02 de maio somavam-se 1.666 casos confirmados e 65 óbitos em 145 municípios. No correr desses 53 
dias, a dinâmica espacial do evento experimentou processos de concentração e difusão que devem ser compreendidas e são relevantes para as políticas de vigilância nas próximas semanas e meses.

O mapa da Figura 1 demostra esses processos simultâneos, que é crescimento do número de casos e sua extensão, em movimento progressivamente ascendente. $\mathrm{O}$ que alguns países fizeram para conter a epidemia foi adotar estratégias para reduzir o número de áreas (municípios, cidades, bairros, regiões) acometidas, pois na medida em que elas aumentam em número, aumentam igualmente os desafios de contenção, devido a própria extensão geográfica do fenômeno. A China foi a primeira a fazer isso e com estratégias próprias da sua realidade política, simplesmente fechou uma cidade inteira para conter o vírus. A Itália também tentou fechar ou conter sua área mais atingida, a região de Lombardia, mas não obteve o mesmo sucesso. É importante compreender o seguinte: o vírus se concentra sempre mais onde há maior densidade demográfica. Mas ele também se difunde a partir de nucleamentos principais, verdadeiras células, e vai ganhando extensão. O problema é que na medida em que se estende, aumentam os focos difusores, que funcionam como novos nucleamentos que vão desempenhar o mesmo papel, num processo vicioso que, salvo medidas de contenção, se generaliza e pode alcançar mesmo áreas e comunidades remotas.

Figura 1 - Números e densidade de casos de COVID-19, por semana epidemiológica, contadas entre 10 de março e 03 de maio de 2020, Rio Grande do Sul, Brasil

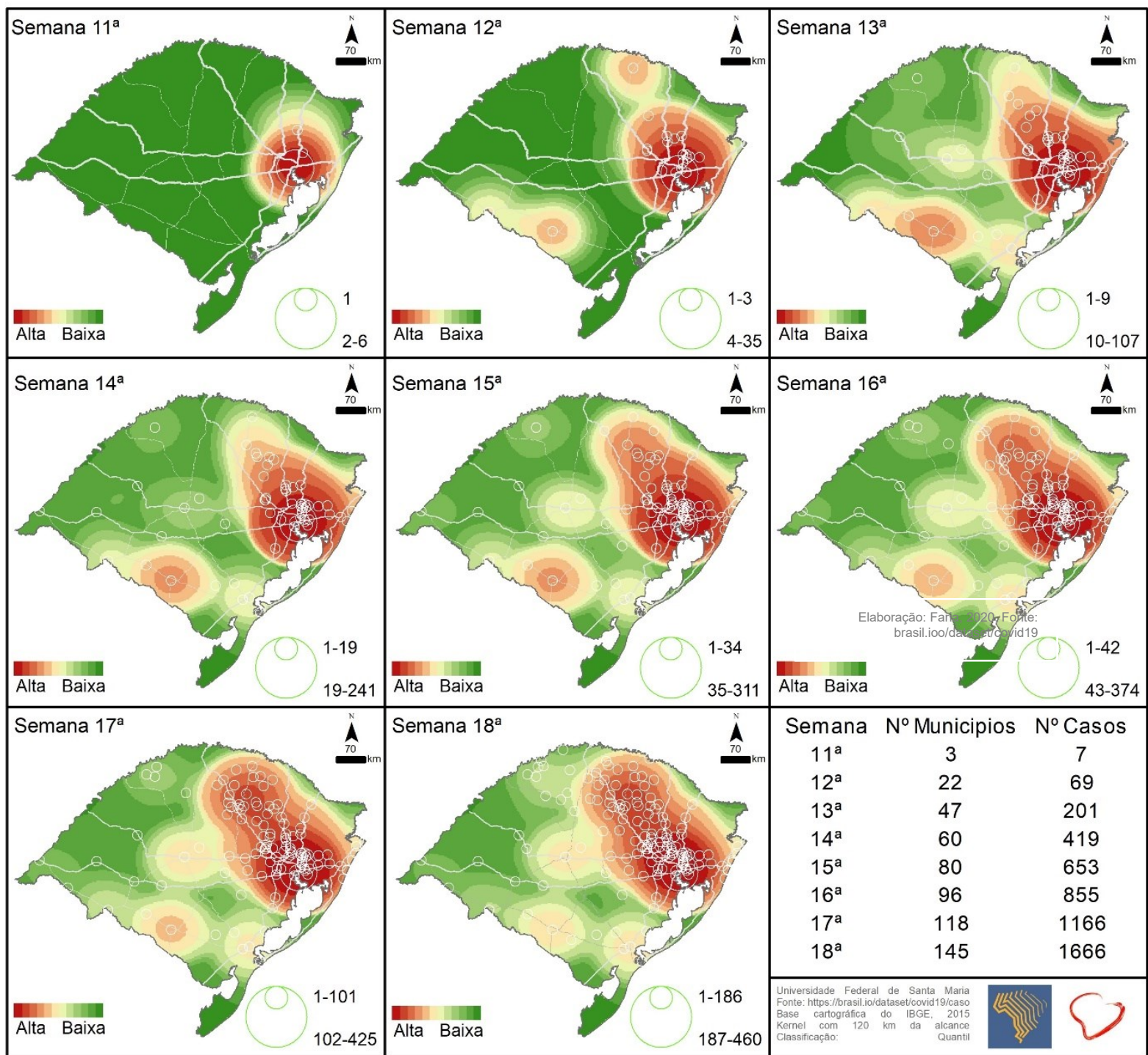

Elaboração: FARIA, 2020 
Um dos aprendizados mais importantes, portanto, é evitar a generalização do evento, ou seja, sua difusão para todos ou quase todos os municípios do estado do Rio Grande do Sul, uma vez que isso vai exigir ações muito mais amplas e processos decorrentes que podem ser imprevisíveis. $O$ que preocupa no Rio Grande do Sul, como no Brasil em geral, é a rápida expansão e a consequente extensão espacial dos casos. Em oito semanas epidemiológicas, o vírus já é registrado desde Porto Alegre aos municípios remotos das fronteiras oeste e sul. Consideremos essa dinâmica espacial, que pode ajudar a pensar ações de vigilância e dimensionamento da possível carga sobre o sistema de saúde.

Como era de esperar e como vem ocorrendo em todos os locais do mundo, a COVID-19 se estende pela rede urbana das cidades maiores às menores em processos que podem ser tanto mais rápidos ou mais lentos, a depender das características da própria rede, das decisões tomadas pelos órgãos de controle social e da dinâmica dos fluxos. Por óbvio, como a COVID-19 é transmitida pelo contato, maiores densidades e maiores fluxos tendem a facilitar sua propagação. Ainda na China, um primeiro estudo, evidenciou esse comportamento (KRAEMER et al, 2020).

Portanto, seguindo padrão mundial e brasileiro, os primeiros casos de COVID-19 ocorreram na Região Metropolitana de Porto Alegre (RMPA), estenderam-se, em seguida, para o Região Metropolitana da Serra Gaúcha (RMSG), tendo Caxias do Sul como sua cidade principal, e até a terceira semana $\left(13^{a}\right)$ já se tinha registrado casos do litoral norte ao sul (de Torres à Pelotas e Rio Grande) e em cidades médias do interior importantes como Santa Maria (centro do estado), Passo Fundo e Erechim (ao norte) e Bagé (fronteira sul). E através desses nós da rede urbana, ainda em março, já se encontravam casos registrados em cidades como em Santa Rosa (fronteira oeste), Santigo e Nova Palma (ao centro), Sant'Ana do Livramento e Dom Pedrito (fronteira sul), entre outras cidades menores localizadas ainda sob influência das duas regiões metropolitanas. Qualquer inspeção rápida no mapa da Figura 2 permite mesmo dizer que a COVID-19 segue o modelo rodoviário sul-rio-grandense, sobretudo os eixos conectados pela BR-101, da RMPA ao litoral sul e norte; a BR-116, na direção da Serra Gaúcha; a BR-386 que liga a capital ao noroeste do estado; a BR-287, na direção oeste, passando por Santa Maria, e, finalmente, a BR-290 que liga a capital a fronteira oeste (Uruguaiana), entre outras, como a BR-153, que conecta as cidades da fronteira sul.

A difusão dos casos e sua densificação (Figura 1 e 2) permite facilmente reconhecer duas principais direções da expansão da COVID-19 no estado Rio Grande do Sul: na rede urbana que vai de Porto Alegre à serra gaúcha, que é a rede de maior densidade e de maior fluxo, e na rede que vai na direção da campanha, que é uma rede urbana de menor densificação e fluxo, mais antiga e, também, mais fragmentada. Por essa razão, é maior o número de casos na rede urbana da serra, estendendo-se, por conseguinte, até Passo Fundo e Erechim. Essa constatação óbvia foi reconhecida em trabalho divulgado por colegas da geografia de Pelotas (DUARTE et al, 2020). Como também é óbvio que a COVID-19 experimenta processos de concentração, sempre maior em locais de elevadas densidades populacionais.

A região metropolitana de Porto Alegre, é, naturalmente, o principal objeto de concentração e difusão da COVID-19 no estado, resultado do seu papel político e econômico. Entre a capital e Caxias do Sul, forma-se a primeira célula do COVID-19 (figura 2), uma ampla área que concentrava, até o dia deste trabalho, $68,1 \%$ do total de casos. Trata-se da região mais populosa e desenvolvida do estado, envolvendo tanto a economia industrial, o turismo e a economia agrária moderna e da pequena produção. A segunda célula é um prolongamento da primeira, ainda envolvendo a região mais desenvolvida do estado, tendo Passo Fundo como seu polo irradiador, abarcando inúmeros municípios com casos confirmados ao seu redor e estendendo-se até Erechim. Essa célula concentra $21,8 \%$ dos casos do estado e nos últimos dias, foi onde se registrou, proporcionalmente, o maior aumento do número de casos novos. Portanto, mais de $89,9 \%$ dos casos de COVID-9 registrados no Rio Grande do Sul estão concentrados no eixo que vai da capital à serra e se estende até o norte do estado.

A mortalidade é uma consequência do maior número de infectados nessas duas grandes células da COVID-19. Até 02 de maio foram registrados 65 óbitos e 55 deles $(84,6 \%)$ ocorreram nesse mesmo eixo urbano e regional. 
Figura 2 - Densidade e distribuição espacial de casos de COVID-19 no estado do Rio Grande do Sul, Brasil, em 02 de maio de 2020 ( $18^{\mathrm{a}}$ semana epidemiológica).

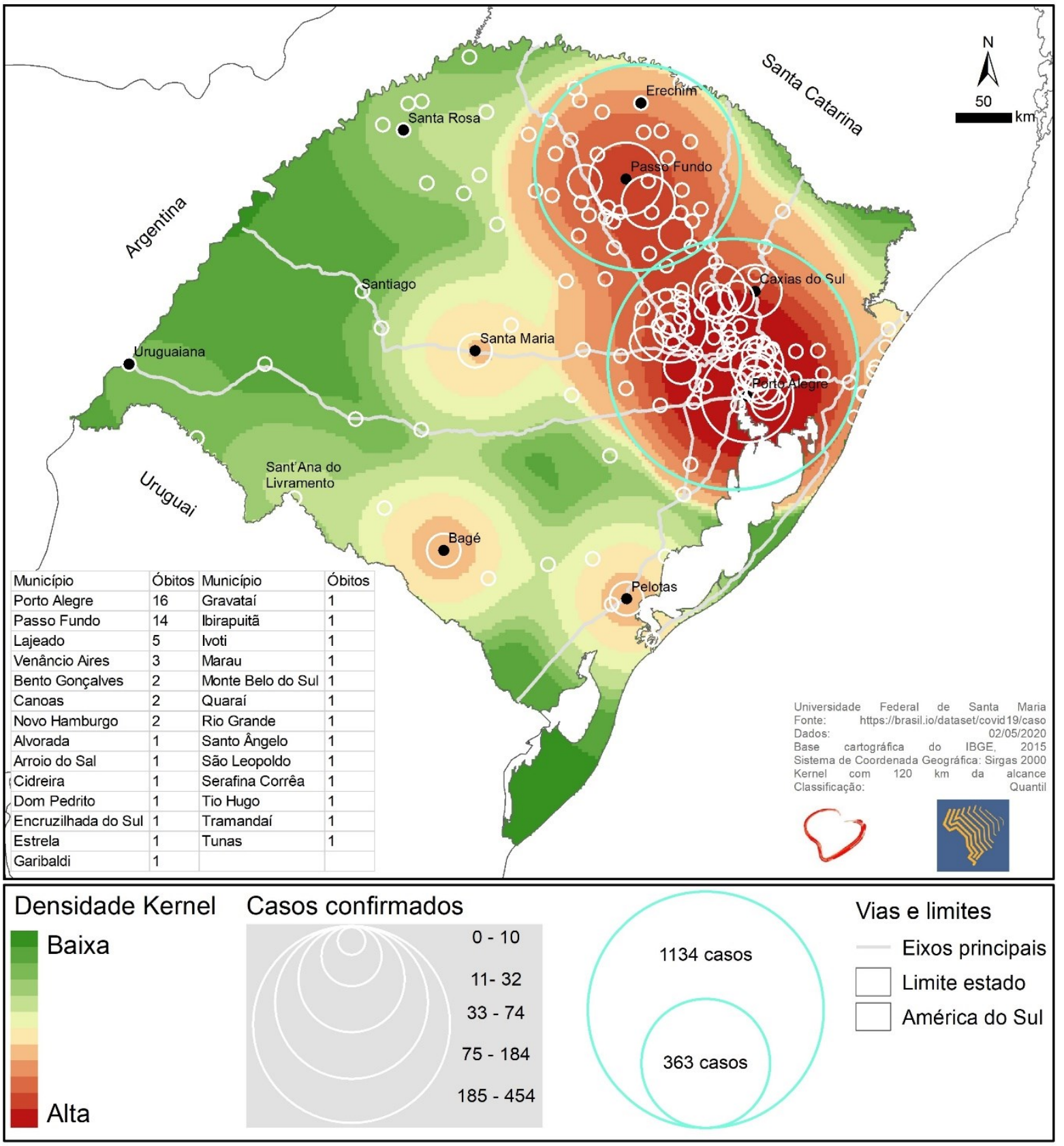

Elaboração: FARIA, 2020

É recomendado pensar, portanto, essas duas grandes áreas de concentração da COVID-19 como uma unidade espacial que carrega características territoriais próprias que explicam a maior disseminação do vírus no estado. O Rio Grande do Sul, como o Brasil, em geral, é conhecido pelas profundas desigualdades territoriais. A dispersão da COVID-19 pela rede urbana, praticamente obedecendo a seu modelo hierárquico, ocorre naturalmente não apenas nos locais de maior adensamento humano, mas também de maior fluidez. E a fluidez, que é um conceito caro para a geografia, é resultado de processos sociais e históricos, estando ligado à realidade social do presente e, principalmente, aos processos econômicos que conectam o Rio Grande do Sul à sua capital, ao Brasil e ao mundo. Por isso, a concentração espacial de casos no eixo "Porto Alegre-Serra-Norte gaúcho" segue exatamente o traçado da região mais desenvolvida do estado. 
Esse parece ser um comportamento geográfico do vírus, sempre se instalando e se difundindo das regiões mais desenvolvidas às menos desenvolvidas. Razão porque, para citar um exemplo, os primeiros casos registrados na Itália, ocorreram ao norte, reconhecidamente sua região mais desenvolvida. Assim também o é em São Paulo, Rio de Janeiro e, claro, no Rio Grande do Sul. Essa compreensão é bastante importante, pois o grande desafio é quando o vírus chega na periferia das cidades e nos locais de menor fluidez e menos desenvolvidos do território. Observe-se na Figura 3 que as áreas com menor Índice de Privação Social (IPS) são também as que possuem a maior densidade de casos registrados de COVID-19, nomeadamente no eixo entre Porto Alegre e Passo Fundo. Mas mesmo no interior, o que se observa é maior número de casos nos municípios socialmente menos privados. É o caso de Santa Maria, Pelotas, Santa Rosa, entre outros.

Figura 3 - Índice de Privação Social (IPS) e distribuição espacial dos casos de COVID-19 por municípios do estado do Rio Grande do Sul, Brasil, em 02 de maio de 2020.

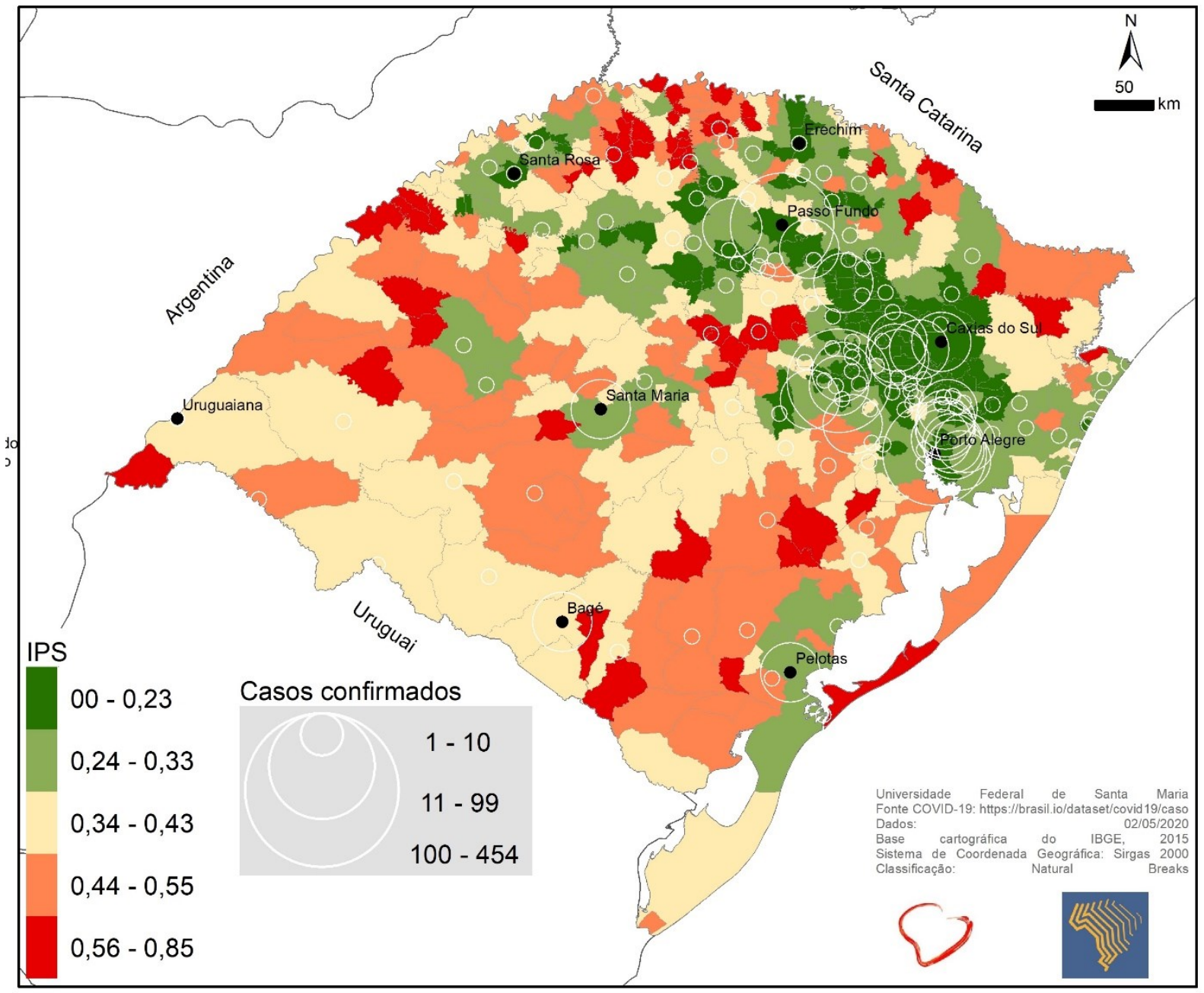

Elaboração: FARIA, 2020

Podemos pensar a geografia da COVID-19 em relação aos níveis da privação social no Rio Grande do Sul, isso para se ter uma ideia do cenário atual da difusão. Até agora, nesse momento de transmissão sustentada, o vírus está mais densamente presente nos municípios com melhores condições sociais. $77,5 \%$ dos casos foram registrados em municípios de baixa privação social $(\leq 0,23)$ (tabela 1$)$. Se considerarmos os dois primeiros intervalos de corte, no que estamos designando de baixa privação social $(\leq 0,33)$, mais de $93 \%$ dos casos registrados até dia 02 de maio estão concentrados nesses municípios (onde está presente $67,2 \%$ da população do estado). Apenas 28 casos de COVID-19 
(menos de 1,6\% do total) foram registrados em municípios de alta privação social (onde reside 9,8\% da população do estado).

Tabela 2 - Classificação dos casos de COVID-19 por níveis de privação social dos municípios do Rio Grande do Sul, Brasil

\begin{tabular}{cccccc}
\hline Intervalos & $\begin{array}{c}\text { Níveis de } \\
\text { privação social }\end{array}$ & $\begin{array}{c}\text { No Casos } \\
\text { COVID-19 }\end{array}$ & $\begin{array}{c}\text { Proporção casos } \\
\text { COVID-19 (em \%) }\end{array}$ & $\begin{array}{c}\text { População } \\
\text { residente }\end{array}$ & $\begin{array}{c}\text { Proporção da população } \\
\text { residente (\%) }\end{array}$ \\
\hline $00-0,23$ & Muito baixa & 1294 & 77,7 & 4706052 & 41,4 \\
\hline $024-033$ & Baixa & 268 & 16,1 & 2940740 & 25,8 \\
\hline $034-043$ & Média & 76 & 4,6 & 875846 & 7,7 \\
\hline $044-055$ & Alta & 22 & 1,3 & 244134 & 2,1 \\
\hline $056-085$ & Muito alta & 6 & 0,4 & 875846 & 7,7 \\
\hline
\end{tabular}

Fonte: https://brasil.io/dataset/covid19/caso / www.ibge.gov.br

Elaboração: FARIA, 2020

O que se deve alertar aqui é o seguinte: até o presente momento, pelos dados deste estudo, a "equação" da epidemia se deu pela relação "COVID-19 e áreas de adensamento populacional mais desenvolvidas". Uma outra equação deve ser fortemente evitada, qual seja: "COVID-19 e áreas rarefeitas de elevada privação social". E pelo mapa da Figura 3 podemos indicar que essas áreas ou municípios estão, principalmente no Pampa e nas fronteiras sul e oeste do estado. Imagine-se uma pessoa com COVID-19 na área rural de município afastado da fronteira e há horas, ou até mesmo dias distante de uma cidade com unidade de referência para tratamento da doença.

Há três sinais sintomáticos da interiorização da COVID-19 no Rio Grande do Sul e já o que podemos afirmar como o principiar do seu alcance nos municípios mais privados de condições econômicas e de saúde. O primeiro movimento se dá no extremo noroeste e oeste do estado, como se pode observar pelos casos na região de Santa Rosa e em municípios pequenos e remotos, como Derrubadas, para citar um exemplo. Isso é resultado de um eixo de difusão que ocorre na região de Passo Fundo e vai se estendendo por municípios do interior. O segundo movimento parece ocorrer entre Porto Alegre e Santa Maria, tendo Lajeado, Venâncio Aires e Santa Cruz do Sul como entreposto. Pelo mapa da densidade da Figura 2, trata-se de um densificação em desenvolvimento, podendo ou não se aprofundar. O terceiro, finalmente, é o eixo que conecta a rede urbana sul, que vai de Pelotas à Bagé e Uruguaiana, perfazendo toda a fronteira. Bagé assustou pelo volume de casos logo no mês de março, mas os dados parecem ter se estabilizado em fins de abril. Mesmo assim, é uma situação que, dada a complexidade do evento, pode ser relativamente imprevisível, haja vista ainda a região do Pampa vem aumentando casos isolados em municípios como São Gabriel, Alegrete, Rosário do Sul, entre outros.

A questão que se coloca é que esses dois possíveis eixos de expansão conectam a COVID-19 com os municípios socialmente mais privados de condições e, por conseguinte, com maiores necessidades de saúde. Novamente devemos recordar que quanto maior a extensão espacial do evento, maiores são as dificuldades de controle e, possivelmente, maiores as consequências para a saúde da população.

\section{CONSIDERAÇÕES FINAIS}

Em observância aos dados apontados nesse artigo, o estado do Rio Grande do Sul ainda se encontra em processo de ascensão dos casos confirmados e de cidades abrangidas pelos vírus. Nesse sentido, é perceptível que ainda não se chegou ao pico da difusão da COVID-19, no estado, como ocorre com estados de São Paulo, Rio de Janeiro e Amazonas.

Por essa razão, é necessário alertar que os casos tendem a aumentar no decorrer das próximas semanas e meses. As medidas de distanciamento social devem ser mantidas e divulgadas a população, para que se previna a difusão atual e retarde ainda mais a curva de contaminação e, por sua vez, não sobrecarregue o sistema de saúde. Recomendamos, a partir dos achados deste trabalho, medidas específicas para as regiões mais carenciadas do estado, municípios com elevada privação social e com difícil acesso aos serviços de saúde. Há duas questões importantes que se deve pensar. A primeira é 
a própria condição social desses municípios, normalmente associadas a piores condições de saúde. A segunda questão é a dificuldade, dada pela distância, para desenvolver uma logística de transporte de pacientes de municípios pequenos aos municípios populacional de porte grande e médio. A terceira, finalmente, é que a própria locomoção de pacientes acaba sendo fator de risco de contaminação e deterioração da condição de saúde de pessoa adoentada. Por tudo isso, recomenda-se antecipar ações, seja para a prevenção, seja ainda para a possível locomoção de pacientes pelo estado.

\section{AGRADECIMENTOS}

Ao Observatório de Dados da COVID-19 da Universidade Federal de Santa Maria, Rio Grande do Sul. Ao grupo da "Força Tarefa de Geógrafos da Saúde" criado para investigação da COVID-19 no Brasil.

À Coordenação de Aperfeiçoamento de Pessoal de Nível Superior - Brasil (CAPES) - Código de Financiamento 001.

\section{REFERÊNCIAS}

(NEDUR) da Universidade Federal do Paraná, Curitiba, Abril/2020.BRASIL. Ministério da Saúde. Brasil confirma primeiro caso da doença. 26 fev. 2020. Disponível em $<$ https://www.saude.gov.br/noticias/agencia-saude/46435-brasil-confirma-primeiro-caso-de-novocoronavirus > Acesso em 27 abr. 2020.

BRASIL. Ministério da Saúde. Painel Coronavírus, 2020. Disponível em <https://covid.saude.gov.br/>. Acesso em 27 abr. 2020.

BRASIL. Atlas do desenvolvimento humano no Brasil. Instituto de Pesquisa Econômica e Aplicada - IPEA. 2013.

BRASIL.IO. Repositórios de dados públicos disponibilizados em formato acessível. Especial COVID19: Dados por município. Disponível em: https://brasil.io/home/ Acesso em: 01 de maio de 2020.

CAMARGO, E. C.; FUCKS, S. D.; CÂMARA, G. Análise especial de superficies. In: DRUCK, SUZANA et al (org.). Análise espacial de dados geográficos. Brasília: EMBRAPA; 2004. p. 155-205.

COSTA, H. G. Introdução ao método de análise hierárquica: análise multicritério no auxílio à decisão. / Helder Gomes Costa. - Niterói, RJ: H. G. Costa, 2002.

DUARTE, T. S.; VIEIRA, S. G.; MARZULLO, M.; SILVA, A. C.; QUEIROZ, A. L. K.; SCHUMANN, E. COVID - 19: Estudos Geográficos. Principais rotas de dispersão do vírus no Rio Grande do Sul. 1804-2020. Disponível em: https://wp.ufpel.edu.br/cidadeecidadania/2020/04/18/grupo-de-pesquisacovid-19-estudos-geograficos-leur-ufpel-realiza-mapeamento-das-principais-rotas-de-dispersao-dovirus-no-rio-grande-do-sul/. Acesso em: 06 de Maio 2020.

Econômicos do COVID-19 no Brasil. Nota Técnica NEDUR-UFPR

FARIAS, H.S. O avanço da covid-19 e o isolamento social como estratégia para redução da vulnerabilidade. Rev. brasileira de geografia e economia. n. 17,12 p. 2020. https://doi.org/10.4000/espacoeconomia.11357

GETIS, A.; ORD, J.K.. The analysis of spatial association by use of distance statistics. Geographical Analysis, 24:-206, 1992. https://doi.org/10.1111/j.1538-4632.1992.tb00261.x

GUAN, W.; NI, Z.; HU, Y.; LIANG, W. H. Clinical Characteristics of Coronavirus Disease 2019 in China. The New England Journal of Medicine. London, 2020.

GUIMARÃES, R. B. Geografia e saúde. In:. São Paulo: Editora UNESP, 2015 Saúde: fundamentos de Geografia humana [online]. 2015.

KRAEMER, M. U. G. et al. The effect of human mobility and control measures on the COVID-19 epidemic in China.

KRAEMER, M.U. et al. The effect of human mobility and control measures on the Covid-19 epidemic in China. Science [Internet]. 2020 Mar [cited 2020 Mar 27]:eabb4218. Available from: Available from: https://science.sciencemag.org/content/early/2020/03/25/science.abb4218

LI, SW, LIN, C.W. Human coronaviruses: Clinical features and phylogenetic analysis. BioMedicice, v. 3, n.1, p. 43-50, 2013. https://doi.org/10.1016/j.biomed.2012.12.007 
OPAS. Organização Pan-Americana de Saúde. OMS declara emergência de saúde pública de importância internacional por surto de novo coronavírus. 2020, Disponível em: $<$ https://www.paho.org/bra/index.php?option=com_content\&view=article\&id=6100:oms-declaraemergencia-de-saude-publica-de-importancia-internacional-em-relacao-a-novocoronavirus\&ltemid=812> Acesso em 27 abr. 2020.

Porsse, A. A.; Souza, K. B. de; Carvalho, T. S.; Vale, V. A. Impactos

PORSSE, A.A.; SOUZA, K.B.; CARVALHO, T.S.; VALE, V.A. Impactos econômicos do covid-19 no Brasil. Nota técnica NEDUR-UFPR. n 01-2020, Núcleo de Estudos em Desenvolvimento Urbano e Regional (NEDUR) da Universidade Federal do Paraná, Curitiba, Abril/2020.

RIO GRANDE DO SUL. Secretaria de Estado de Saúde. Confirmado o primeiro caso de novo coronavírus no Rio Grande do Sul, 2020. Disponível em <https://saude.rs.gov.br/confirmado-oprimeiro-caso-de-novo-coronavirus-no-rio-grande-do-sul> Acesso em 02 mai. 2020.

SAATY, T.L., How to make a decision: The analytic hierarchy process. European Journal of Operational Research, Vol.48 No.1, pp.9-26, 1990. https://doi.org/10.1016/0377-2217(90)90057-I

SANTOS, M. A natureza do espaço: técnica e tempo, razão e emoção. 2. reimpr. São Paulo: Editora da Universidade de São Paulo, v. 1, 2006.

SORRE, M. Fundamentos da geografia humana. Max. Sorre, Ática, São Paulo, p. 87-98, 1984.

SPOSITO, M. E. B.; GUIMARÃES, R. B. Por que a circulação de pessoas tem peso na difusão da pandemia. Difusão da Covid-19 no país segue modelo relacionado a interações espaciais na rede urbana. por: Maria Encarnação Beltrão Sposito e Raul Borges Guimarães. 26/03/2020. UNESP. Universidade Estadual Paulista "Júlio de Mesquita Filho". Disponível em: https://www2.unesp.br/portal\#!/noticia/35626/por-que-a-circulacao-de-pessoas-tem-peso-na-difusaoda-pandemia. Acesso em: 10 Abr. 2020.

WHO. World Health Organization. Coronavirus disease (COVID-19) outbreak. Genebra: WHO, 2020. Disponivel em: https://www.who.int/dg/speeches/detail/who-director-general-s-opening-remarks-at-themedia-briefing-on-covid-19---11-march-2020 Acesso em 27 abr. 2020.

VOOGD, H. Multicriteria evaluation for urban and regional planning. London: Pion, 1983. 